\title{
Commentary on 'Can effective supervised pelvic floor muscle training be provided by primary care nurses? A randomized controlled trial'
}

\author{
Abdelmageed Abdelrahman ${ }^{1}$ \\ Received: 25 January 2021 / Accepted: 6 March 2021 / Published online: 15 May 2021 \\ (C) The International Urogynecological Association 2021
}

This randomized controlled trial (RCT) aimed to determine whether primary care nurses with no prior experience can, after training, provide effective supervised pelvic floor muscle training (PFMT). The trial had three groups, primary care nurse, urogynaecology nurse specialist and controls, and was undertaken in 11 primary care/general practices, covering urban and rural settings in South West England.

A total of 240 women were recruited. The primary outcome used for effectiveness of PFMT was pelvic floor muscle strength assessed by perinometry. After 3 months, there was an increase in strength in both intervention groups compared with controls. Also of note, there was no difference between the primary care nurse and urogynaecology nurse specialist groups. The authors concluded that PFMT provided by trained primary care nurses achieved improvements in pelvic floor muscle strength compared with controls. This was also comparable to that of a urogynaecology nurse specialist.

The authors present an interesting study that could have implications for the provision of PFMT worldwide. This is the first RCT to provide level 1 evidence that PFMT delivered by a primary care nurse following training can produce a significant improvement in pelvic floor muscle strength. Limitations of the study include it being undertaken between 2003 and 2005. Also of note, during the course of the study, there was no 'gold standard' regimen of PFMT; therefore, the authors used a regimen that was consistent with other RCTs and recommendations at the time. Perineometry is not recommended in routine practice; however, it was used for this RCT as it has been shown to have satisfactory reliability. There was a drop-out rate of $34 \%$, suggesting that women may not adhere to such a programme.

\section{Declarations}

Conflict of interest None.

Publisher's note Springer Nature remains neutral with regard to jurisdictional claims in published maps and institutional affiliations.
Abdelmageed Abdelrahman

abdelmageed@hotmail.co.uk

1 Department of Urogynaecology, Liverpool Women's Hospital NHS Foundation Trust, Liverpool, UK 